\title{
Quantitative sensory testing in atypical odontalgia patients after local anesthesia
}

\author{
AL Porporatti', PCR Conti \\ From The European Headache and Migraine Trust International Congress \\ London, UK. 20-23 September 2012
}

\section{Introduction}

Atypical Odontalgia (AO) is a term applied to a continuous pain in the teeth or in the tooth socket after extraction in the absence of any identifiable dental cause, under the International Classification of Headache Disorders (ICHD) and somatosensory abnormalities are common features in these patients, where Quantitative Sensory Testing (QST) are helpful tools to evaluate these cases.

\section{Objectives}

The aim of the pilot study was to determine the effect of topical application of anesthetic cream in quantitative sensory testing (QST) findings of AO patients.

Methods Ten ( 7 women and 3 men) consecutive AO patients $(60 \pm 14,97$ years $)$ with mean pain duration of 2.7 years (range 1-10 years were recruited from Bauru School of Dentistry, University of São Paulo (Brazil). QST was performed in all patients at baseline and 3 minutes after topical application of anesthetic cream (Benzocaine $2 \%$ - Benzotop $200 \mathrm{mg} / \mathrm{g}, \mathrm{DFL})$. QST included tests of mechanical detection threshold (MDT) and mechanical pain threshold (MPT) with von Frey monofilaments, dynamic mechanical allodynia with cotton swab (DMA1) and with toothbrush (DMA2), heat pain thermal detection (HPD), cold pain thermal detection (CPD), temporal summation (WUR) and controlled pain modulation (CPM). The present pain intensity was also recorded with visual analogue scale (VAS). Results were analyzed with nonparametric Wilcoxon test with significance level of $5 \%$.

\section{Results}

QST mean values showed no differences after topical application of anesthetic cream, except for DMA2 $(\mathrm{p}=0.02)$ and WUR $(\mathrm{p}=0.02)$. Moreover mean pain in visual analogue scale relieved from 6,03 to $2,12(64,84 \%$; $\mathrm{p}=0.02$ ).

\section{Conclusion}

In this pilot study significant changes in intraoral somatosensory function were observed in $\mathrm{AO}$ after topical application of anesthetic cream for dynamic mechanical allodynia, associated to a reduction of pain intensity. These results may reflect peripheral and central sensitization of trigeminal pathways.

\section{Conflict of interest}

There were no conficts of interest in the performance of this study.

Published: 21 February 2013

References

1. Olesen J, Steiner TJ: The International classification of headache disorders, 2nd edn (ICDH-II). J Neurol Neurosurg Psychiatry 2004, 75(6):808-811.

2. Svensson P, Baad-Hansen L, Pigg M, List T, Eliav E, Ettlin D, Michelotti A Tsukiyama Y, Matsuka Y, Jaaskelainen SK, Essick G, Greenspan JD, Drangsholt M, Special Interest Group of Oro-facial P: Guidelines and recommendations for assessment of somatosensory function in orofacial pain conditions-a taskforce report. J Oral Rehabil 2011, 38(5):366-394.

doi:10.1186/1129-2377-14-S1-P159

Cite this article as: Porporatti and Conti: Quantitative sensory testing in atypical odontalgia patients after local anesthesia. The Journal of Headache and Pain 2013 14(Suppl 1):P159. 